Horizons philosophiques

\title{
Existence malheureuse et temporalité
}

\section{Réflexions kierkegaardiennes sur le sens de l'existence}

\section{Dominic Desroches}

Volume 14, numéro 1, automne 2003

$\mathrm{Au}$ risque du bonheur

URI : https://id.erudit.org/iderudit/801250ar

DOI : https://doi.org/10.7202/801250ar

Aller au sommaire du numéro

Éditeur(s)

Collège Édouard-Montpetit

ISSN

1181-9227 (imprimé)

1920-2954 (numérique)

Découvrir la revue

Citer cet article

Desroches, D. (2003). Existence malheureuse et temporalité : réflexions kierkegaardiennes sur le sens de l'existence. Horizons philosophiques, 14(1),

39-55. https://doi.org/10.7202/801250ar d'utilisation que vous pouvez consulter en ligne.

https://apropos.erudit.org/fr/usagers/politique-dutilisation/ 


\section{EXISTENCE MALHEUREUSE ET TEMPORALITÉ}

\section{RÉFLEXIONS \\ KIERKEGAARDIENNES SUR LE SENS DE L'EXISTENCE}

Kierkegaard est un penseur de l'existence. Or face à cette question difficile qui consiste à "trouver" le sens de l'existence, la démarche philosophique de Kierkegaard est originale et peut nous servir de guide. Celle-ci consiste à proposer une topologie existentielle au sein de laquelle s'exposent et s'illustrent les grandes catégories qui permettent à tous, en tant qu'individus, de "se comprendre dans l'existence ${ }^{1}$ ». II y a trois sphères ou stades de l'existence dans la philosophie de Kierkegaard : l'esthétique, l'éthique et le religieux. Et c'est le langage, dans les limites de ce qu'il peut dire, qui marque la différence entre ces trois conceptions de vie : entre la deux premières sphères se trouve l'ironie, qui consiste à faire entendre quelque chose par son contraire, et entre les deux dernières se trouve l'humour, qui consiste à reconnaître les limites de son existence finie face à l'infini2. Si toute pensée qui se penche sur le sens de l'existence ne peut manquer de soulever la question du bonheur et du malheur, celle du philosophe danois, aussi, n'y échappe pas.

Kierkegaard distingue la joie (Glæde), la félicité éternelle (evige Salighed) et le bonheur (Lykke). Si le bonheur constitue le sentiment propre à la sphère esthétique qui, face aux sphères éthique et religieuse, ne s'est pas encore remise en question elle-même (c'est la critique que l'on peut lire dans le second tome du Post-Scriptum aux Miettes philosophiques), Kierkegaard fait en revanche de la joie durable et de la félicité des sentiments religieux, comme la joie et le salut propres au christianisme. En fait, on ne peut accéder à la félicité sans le christianisme. Le bonheur ne peut se comprendre ici qu'à la lumière de la souffrance de l'intériorité éthique et religieuse ${ }^{3}$. Ainsi nous avons, en résumé, le schéma traditionnel de la topologie kierkegaardienne appliqué à la question du bonheur. 
Dans les pages qui suivent, nous voulons nuancer cette lecture très commode et reprendre à nouveaux frais la question du bonheur chez Kierkegaard, car nous croyons qu'il n'est pas vain d'étudier le bonheur hors du cadre religieux. Notre intérêt n'ira donc pas à la joie et à la félicité éternelle, des notions religieuses développées dans le cadre du problème de la souffrance dans le Post-scriptum, les Discours édifiants (1844) et les Discours chrétiens $(1848)^{4}$, qui ont déjà été étudiées ailleurs 5 , mais au malheur (ulykke) compris comme conflit propre à la personnalité esthétique. C'est ainsi que nous nous attaquerons au bonheur par la voie négative, c'est-à-dire en approfondissant le problème du malheur. Partant du malheur, notre méthode ne surprendra personne tellement elle est classique en philosophie, car plus souvent qu'autrement, les philosophes, incapables de dire en quoi consistait le bonheur, se sont limités à déduire les caractéristiques du bonheur à partir de la description de son contraire, c'est-à-dire du malheur.

Dans cet article, nous montrerons que la question du malheur (donc aussi celle du bonheur) est inséparable chez Kierkegaard de l'interprétation du temps. Nous défendrons la thèse suivant laquelle l'éthique, grâce à la notion de continuité de soi, assure à la fois un sens à l'existence, mais aussi une certaine stabilité, un certain bonheur. Après avoir présenté la sphère esthétique, expliqué trois formes de malheur liées à des interprétations inadéquates de la temporalité et montré en quoi la continuité éthique permet de découvrir un sens à l'existence, nous insisterons sur la différence absolue séparant le bonheur et le malheur dans l'interprétation du sens de l'existence. Cela nous permettra de voir comment la pensée de Kierkegaard se démarque de la tradition philosophique pour laquelle le bonheur, le plus souvent, est à entendre comme «bonne fortune". À la fin de notre article, nous nous pencherons, dans une perspective herméneutique, sur le bonheur et la question du sens de la vie. Pour Kierkegaard, nous le verrons, il n'est pas tant question de savoir si le bonheur est le but ultime de l'existence, de savoir s'il se trouve au hasard de la vie ou si nous en sommes dignes, comme le proposait Kant, mais plutôt de chercher à comprendre de quelle manière l'homme peut travailler sur lui-même afin d'éviter, ici-bas, le malheur dont il est lui-même responsable. 


\section{Mode esthétique d'existence : immédiateté, paradoxe et vide}

Pour Kierkegaard, l'esthétique n'est pas d'abord une science ou un savoir, mais un mode d'existence immédiatement déterminé comme désir et imagination en l'absence de l'unité du moi. L'esthétique s'avère une sphère en laquelle l'existence est caractérisée par la recherche immédiate de la sensation, du plaisir et de l'atmosphère. Voilà pourquoi Kierkegaard déclare dans la première partie de Enten-Eller (L'Alternative) consacrée à l'exposition de la sphère esthétique : est esthétique "ce par quoi l'on est immédiatement ce que l'on est" (OC IV, 162 / II 193). Or cette définition de l'esthétique en tant que modalité d'existence n'est pas habituelle en philosophie et elle mérite des explications.

L'esthétique se veut la sphère de la multiplicité, du possible, de la discontinuité. Elle incarne d'abord la multiplicité parce qu'elle s'exprime de multiples manières dans son œuvre : selon plusieurs auteurs, dans plusieurs textes et par de nombreuses figures légendaires. Ensuite, elle est la sphère du possible parce que l'imagination, le moteur de l'esthétique, fournit des possibilités idéales d'existence. De la discontinuité enfin parce que son représentant, sans cesse dominé par ses états d'âme et la recherche du plaisir, ne cherche que la jouissance en des instants épars. Dans la topologie existentielle, la sphère esthétique se présente donc comme une "poétisation romantique du monde" et trouve dans l'élément sensoriel son atmosphère, qui est son seul point de contact : le réel.

Afin de mieux saisir cela, procédons à une petite phénoménologie de l'esthétique. Sous l'emprise partielle sinon totale de son humeur (ou tonalités affectives / Stemninger), la personnalité esthétique se cherche sans cesse. Ballottée au rythme de ses désirs et de ses pulsions, elle est pour ainsi dire forcée de vivre dans la dispersion de ses états d'âme, esclave qu'elle est de sa recherche infinie de la nouveauté et des sensations fortes. Ainsi n'est-elle jamais elle-même, vraie ou authentique, elle se retrouve toujours dans un nouveau rôle, aussi nombreuse que les personnages qu'elle se plaît à jouer! Tout ce qui l'intéresse, c'est l'immédiateté, la sensation et le plaisir. Ainsi vue, la vie esthétique procure le bonheur dans la sensation présente et la gratification instantanée - le malheur lui est étranger, trop sérieux et trop souffrant. La personnalité de l'esthétique ne vise donc que ce bonheur immédiat, accessible au sens et à l'imagination. 
Or, l'esthétique connaît aussi le conflit. En effet, l'esthéticien, le spécialiste de l'immédiat, souffre d'un manque d'unité, car son moi n'est pas construit sur de véritables choix. L'esthétique s'avèrera contradictoire parce qu'elle est vouée exclusivement à la quête de l'idéalité. C'est ainsi qu'elle incarne un danger, car son défenseur apparaît tôt ou tard incapable de sortir du monde des possibles qu'il a lui-même imaginé. II fait de sa vie un théâtre et trouve peu intéressante la vie concrète puisque, face au royaume des idées, celle-ci offre bien peu de satisfaction pour sa féconde imagination.

Ainsi en est-il de la personnalité esthétique : entièrement soumise aux désirs et à l'imagination, elle apparaît fictive parce qu'elle manque d'une véritable passion d'exister. Elle se contredit elle-même, car elle cherche dans la réalité une idéalité qui ne s'y trouve pas et dans l'idéalité une réalité qui la contredit. Le paradoxe propre au mode esthétique d'existence s'établit sur la contradiction qu'il y a à chercher l'idéalité pour elle-même et à s'y abîmer, oubliant par là l'existence concrète, c'est-à-dire la vie éthique (Det Sædelige). La passion d'exister, d'exister dans le temps, affirme Kierkegaard, ne peut se trouver que dans la sphère éthique où l'homme se reconnaît lui-même à partir de ses choix. Tourné vers les plaisirs extérieurs et le monde imaginaire, l'esthète plane dans un ciel splendide, évite les engagements, se dit l'ami du destin, n'en a que pour le rêve et nie l'existence du malheur. Ainsi comprise, l'esthétique s'avère une expérience sans durée, sans continuité : elle aspire à la jouissance dans des moments épars et s'exprime toujours dans un présent vaporeux, dans des instants vides, à l'exclusion des dimensions du passé et du futur.

Face à l'esthétique, l'éthique s'ouvre par la réalité du choix de soi dans le temps. En effet, c'est par "le choix de soi-même" (OC IV, 200-2001 / II 241) donnant sur une continuité, une histoire, que se vit la réalité éthique qu'incarne, dans l'œuvre pseudonyme kierkegaardienne, l'assesseur Wilhelm, le bon père de famille, bourgeois et responsable. Pensée sur la base de la Sittlichkeit hégélienne des Principes de la philosophie du droit6, l'éthique de la conformité sociale n'est jamais l'affaire des humeurs et des caprices, mais elle concerne plutôt le développement de la personnalité, c'est-à-dire la sphère des devoirs. C'est ici la vocation et le mariage qui insèrent l'individu dans le social. 
Nous aimerions revenir à l'esthétique pour soulever à nouveau l'imposture dans laquelle elle plonge l'individu qui s'y adonne exclusivement. L'instant qui la caractérise est subtil : il maintient l'illusion de l'intensité, sans pourtant survivre au déroulement du temps de l'existence. Décrite comme la sphère des états d'âme, lesquels tirent l'individu à l'extérieur de lui-même, dans l'idéal et le rêve, les exigences de l'esthétique conduisent son défenseur dans les vapeurs et l'inertie. Visant le transport de l'âme par l'atmosphère et l'ambiance, il néglige la force du réel et s'engage de moins en moins dans une vie qui ne recèle plus aucun sens pour lui. Les conséquences d'une conception de vie sans unité, sans direction, ne sont pas difficiles à saisir. L'indifférence autant à la vie active qu'aux obligations morales tient l'individu en rupture avec lui-même : isolé, réfuté par ses idéaux et ses rêves impossibles, il tombe dans la mélancolie où l'état d'âme prend définitivement le dessus sur la raison et le devoir. Désintéressé, il se cherche lui-même sans conviction. La vie, désormais, n'a plus de sens pour lui. Il désespère...

Mais que faire devant les sentiments de vide, d'échec et d'ennui? Confronté au désespoir de ne pas être soi-même, on doit certes se demander si l'incapacité à décoder le "sens" de la vie n'indique pas un refus d'assumer le quotidien? Ce nihilisme, terminus de l'esthétique, ne correspond-t-il pas à un déni de l'existence comme tâche (Opgave)? C'est peut-être cette négation du quotidien - des exigences du travail routinier que tout le monde doit assumer qui indique le mieux un manque de continuité dans la personnalité. Car la jouissance immédiate ne peut produire qu'un temps vide, qu'un temps non rempli, qu'une illusion de continuité. Voilà pourquoi l'existence se vivra comme ennui. Souvent d'ailleurs, le souvenir du passé revient comme un fantôme hanter celui qui, cherchant à jouir dans l'instantanéité, a omis de choisir. De même, en sens contraire, l'espoir trouve sa forme dans les idéaux de celui qui, épris de souvenirs, reste prisonnier de lui-même. II est dès lors pour ainsi dire avantageux, sur le plan du sens de l'existence, de se doter d'une certaine "continuité", de manière à ne pas trop souffrir des spectres du passé ou des mirages en l'avenir. C'est que la souffrance provient toujours d'un conflit, d'un moi divisé qui ne se reconnaît pas lui-même. L'esthétique, dit Kierkegaard dans l'Alternative, "échoue au récif du temps". Si l'on ne renverse pas absolument cette conception de l'existence dans l'éthique, par le choix de soi-même dans le temps, on est perdu. 


\section{Le malheur : une question de temporalité ?}

Ou malheureux! Car c'est ainsi que l'on est malheureux soutient Kierkegaard, en n'étant pas présent à soi-même parce que l'on a la tête dans le passé ou dans l'avenir. Le malheureux vit dans des temps qui ne sont pas les siens, pour reprendre ici les mots de Pascal : «Le monde est si inquiet qu'on ne pense presque jamais à la vie présente et à l'instant où l'on vit, mais à celui où l'on vivra. De sorte qu'on est toujours en état de vivre à l'avenir et jamais à vivre maintenant». Ce passage peut très bien être rapproché d'un autre fragment des Pensées, que nous tenons à citer en entier tellement il servira notre propos :

Nous ne tenons jamais au temps présent. Nous nous rappelons le passé ; nous anticipons l'avenir comme trop lent à venir, comme pour hâter son cours, ou nous rappelons le passé pour l'arrêter comme trop prompt, si imprudents que nous errons dans des temps qui ne sont pas les nôtres, et ne pensons point au seul qui nous appartient... Que chacun examine ses pensées. II les trouvera toutes occupées au passé ou à l'avenir. Nous ne pensons presque point au présent, et si nous y pensons ce n'est que pour en prendre la lumière et disposer de l'avenir. Le présent n'est jamais notre fin. Le passé et le présent sont nos moyens; seul l'avenir est notre fin. Ainsi nous ne vivons jamais mais espérons de vivre. (Pensées, Ed. Lafuma, 47)

Ces lignes anticipent sur la pensée de Kierkegaard pour qui la temporalité est une inquiétude ontologique. Pour le dire sans détour : le malheureux est victime de sa propre conception du temps. Est-il dans l'espoir ou dans le souvenir, il n'est jamais dans le présent, donc heureux, car il n'est jamais présent à lui-même dans une pleine continuité. En effet, soit qu'il pense que c'était mieux avant, soit qu'il espère que cela ira mieux après, bref : il n'est jamais en accord avec le temps présent. Kierkegaard, qui connaissait très bien l'œuvre de Pascal (1623-1662) et qui estimait l'auteur des Pensées parce qu'il s'en sentait proche comme chrétien (OC IX, 423), a mis à profit cette manière de voir le malheur dans un très court texte au titre pour le moins évocateur: "Le plus malheureux" (Den Ulykkeligste). Kierkegaard, ce n'est peut-être pas un hasard, développe les idées de Pascal lorsqu'il est précisément question du bonheur, alors que l'on 
sait que Kierkegaard a toujours vu en Pascal un grand chrétien souffrant, un homme malheureux... Pour bien mesurer l'originalité de Kierkegaard en regard de Pascal et de la tradition philosophique, nous analyserons systématiquement ce très court texte, juste après l'avoir situé.

Le texte de seulement huit pages est situé dans la première partie de l'Alternative, entre "Silhouettes" et "Les premières amours». Ainsi précède-t-il le célèbre "Journal du séducteur» qui accomplit le mode esthétique d'existence. Afin de mieux saisir le ton ironique et existentiel du texte, on rappellera que ces méditations sur le malheur, suivant le sous-titre, constituent une "harangue enthousiaste" adressée aux membres d'un cercle privilégié (!), les symparanécroménoï, à savoir le cercle des amis de la mort. Si le nom du cercle a de quoi inquiéter le lecteur, précisons que les méditations se présentent sur fond de concours où l'on cherche à déterminer qui est le "plus malheureux". Sur la question du malheur, l'auteur se permet, au passage, de critiquer la "conscience malheureuse" de Hegel, car si celui-ci a su "fixer des limites", il demeure néanmoins un philosophe qui "contemple les problèmes à distance" (OC III, 208 / I 227). Or, nous avons au moins une clef pour interpréter le texte, il faut s'investir dans l'existence pour cerner le malheur.

Selon notre texte, le malheur n'est pas tant rattaché à la mort qu'à une mauvaise interprétation de la temporalité ou à une mauvaise conception du temps de vie. En effet, c'est la mauvaise interprétation du temps de vie qui conduit à la mort. Le malheureux doit être reconnu dans le fait qu'il n'est pas présent à lui-même dans sa propre vie. Or, l'on peut être absent à soi-même de deux manières : ou bien nous avons la tête dans le passé, ou bien nous avons la tête dans le futur. Ainsi, suivant le critérium de la présence à soi, nous retrouvons deux types de malheureux : celui qui vit dans le passé et celui qui vit dans le futur. Le "plus malheureux", précisera Kierkegaard, sera celui qui vit à la fois dans le souvenir et l'espérance. Dans un passage hautement dialectique, on lit :

Imaginons une combinaison des deux types de malheureux proprement dit que l'on vient d'analyser. L'infortuné du fait de l'espérance ne peut devenir présent à lui-même dans son espérance, et de même l'infortuné du ressouvenir. La combinaison ne peut être que la suivante : ce qui empêche l'individu de devenir présent dans son espérance, c'est le 
ressouvenir; ce qui l'empêche de devenir présent dans son ressouvenir, c'est l'espérance. La raison en est, d'une part, qu'il espère toujours ce dont il devrait se ressouvenir; son espoir étant toujours déçu, il en vient à découvrir la cause de sa désillusion : le but, loin de reculer devant lui, est dépassé; il a été vécu ou aurait dû l'être, et être ainsi entré dans le ressouvenir. D'autre part, l'individu se rappelle sans cesse ce dont il devrait faire l'objet de son espérance, car il a déjà accueilli l'avenir dans sa pensée où il l'a vécu, et il se ressouvient de ce vécu alors qu'il devait l'espérer. Ainsi ce qu'il espère ce trouve derrière lui et ce dont il se souvient, devant lui. Non que sa vie aille à rebours, mais il suit deux directions contraires, toutes deux à rebours. (OCIV, 210-211 / 229-230)

Fort de ce long passage, on pourra dégager trois formes de malheur. La première forme relève du passé : l'individu est malheureux de ne pas être lui-même dans son passé. II peut bien se rappeler les événements de son passé, mais il ne peut s'en souvenir en vivant ses souvenirs puisqu'ils sont passés. L'individu malheureux n'a pas intériorisé les moments épars de son expérience passée et ne peut donc pas s'en souvenir. Le passé lui reste donc fermé. Telle est la première forme de malheur.

La seconde forme de malheur relève de l'avenir : l'individu est malheureux de ne pas être présent à soi-même dans son avenir, à ne pouvoir espérer. Certes, cet avenir est ouvert, contrairement au passé qui est déjà fermé, mais l'individu n'est pas sans savoir que cette ouverture aux possibles ne peut être qu'une ouverture à l'absence, ce qui revient à dire qu'il est identique à une fermeture. L'avenir est possible, mais n'est pas encore réellement possible. En d'autres mots, on doit encore l'attendre! Telle est la deuxième forme de malheur, celle liée à l'impossibilité de vivre immédiatement l'avenir.

La troisième forme de malheur, de loin la plus importante, réside dans la réunion des formes précédentes. Cette réunion peut se "contextualiser" dans l'affirmation suivante : "ce qui empêche l'individu de devenir présent dans son espoir, c'est le souvenir, et ce qui l'empêche de devenir présent dans son souvenir, c'est l'espoir" (OC III, 210 / I 229). Qu'est-ce que cela peut bien vouloir dire? On interprétera ce passage ainsi : l'individu de l'esthétique ne peut être présent à lui-même dans son propre passé parce que les instants 
de plaisir qu'il a vécus n'ont pas pris place dans une expérience passée et n'ont pas été intériorisés. Or, ce qui lui permet d'accepter cette fermeture du passé à sa présence, c'est la découverte que l'avenir est plus proche du présent que le passé. Ainsi misera-t-il sur l'avenir... Mais il n'en sera pas dupe. II ne peut concevoir un avenir différent de son passé, qui est fait d'instants vides d'intériorité. II anticipe ainsi déjà l'évanescence de ces instants futurs, qui ne sont plus accessibles à sa présence, car ils sont déjà passés. Par conséquent, ce qu'il espère se trouve donc derrière lui et ce dont il se souvient plutôt en avant. Selon l'auteur, nous avons ici la forme de malheur du plus malheureux.

Or ces réflexions, peu évidentes à première vue, nous invitent à questionner le rapport de notre tonalité affective à notre conception du temps. Le malheur de l'individu de l'esthétique est de ne pas réussir à se retrouver lui-même dans sa propre expérience du temps. Incapable de bien se situer dans le temps, le temps qui est le sien, désaxé donc, il erre dans l'existence, confondant les moments du passé avec les exigences à venir. Vide apparaît alors l'existence. Telle est la conclusion qu'en tire notre auteur un peu plus bas : "Sa vie n'a aucun sens (...). Sa vie ne connaît pas le repos, elle est vide; il n'est pas présent à lui-même" (Ibia).

\section{Sur le sens de la vie : continuité et contemporanéité}

Ce qui manque à l'homme de l'esthétique, c'est précisément la continuité éthique avec lui-même. Cette continuité est décisive, car elle assure un sens à l'existence. Si elle n'est pas le bonheur, la continuité permet en revanche à l'individu de "trouver» une direction, une harmonie, une stabilité. Voyons ce qu'elle signifie pour l'éthique.

À partir du temps, la continuité ne se présente pas idéalement, mais concrètement. Elle n'est pas l'affaire des mystiques qui cherchent une idéalité hors de l'existence, dans une parousie avec le divin, ni des artistes ou des esthètes qui jouent un rôle dans l'espace public ou dans les salons, mais elle est plutôt la tâche de l'éthique dans la vie ordinaire. Pour Kierkegaard, travailler sur soi-même dans le temps, comme personne ordinaire, en cherchant à donner à son existence finie une continuité, représente rien de moins que la "dignité éternelle de l'homme». Kierkegaard écrit en effet :

La temporalité n'est pas donnée, si j'ose dire, à cause de Dieu, afin qu'il y mette l'amant à l'épreuve comme dit le mystique; 
elle est donnée à cause de l'homme, et de tous les présents de la grâce, elle est le plus grand. L'éternelle dignité de l'homme (Menneskes evige Værdighed), c'est en effet sa capacité d'avoir une histoire, et le divin en lui consiste en ce que, s'il le veut, il peut donner à cette histoire la continuité (hvis kan vil, kan give denne historie Continuitet); car elle possède la continuité seulement quand elle n'est pas un résumé de ce qui est arrivé ou de ce qui arrive, mais est mon action propre, de sorte que même ce qui m'est arrivé se trouve par moi changé et traduit de nécessité en liberté. (OC IV 225 I II 271)

Le choix éthique, qui consiste à se choisir soi-même absolument à l'exclusion de tout élément esthétique, donc de choisir la conception éthique elle-même en nous, permet à l'individu de sortir du relativisme de l'esthétique. En se choisissant lui-même dans le temps, l'individu quitte l'imaginaire pour rejoindre la réalité. II plonge en son existence finie et se retrouve du même coup en continuité avec sa propre réalité. Ainsi assure-t-il un sens à son existence et connaît-il une nouvelle liberté, à savoir celle de se forger sa propre histoire. Pouvons-nous mieux décrire ce qui se passe lorsque l'individu choisit l'éthique, lorsqu'il opte, contre l'esthétique, pour la conception éthique de l'existence? La réponse est affirmative. Pour ce faire, nous préciserons le caractère temporel de ce choix.

Or cette continuité éthique de soi, résultant du choix éthique, n'est pas facile - elle demande des efforts constants, la répétition de la volonté de soi-même. Ainsi, la volonté et l'énergie permettent à l'homme d'actualiser ses choix dans l'existence et, partant, de vaincre les inconstances et les incohérences de l'esthétique. Par ses choix, il se donne une histoire et se "produit» dans le temps. Véritable prise en charge de l'individu par lui-même, "victoire" de l'éthique sur l'esthétique (OCX,235-236 / VII 240), la continuité éthique prend ici toute sa signification. Elle permet à l'individu de dépasser les états d'âme esthétiques, mais aussi d'accepter son rapport au temps dans le temps même car, pour la pensée existentielle en général et pour l'éthique en particulier, le temps doit être envisagé dans un horizon temporel. Ainsi comprise, la continuité éthique s'interprète sur la base d'une expérience éthique personnelle s'enracinant elle-même dans le temps. Comme auto-temporalisation de soi, la vraie continuité procure à l'éthique sa stabilité, mais aussi une possibilité de transfiguration 
(Forklaringelse), car l'individu ne voit plus la vie de la même façon : la vie n'est plus pour lui désespoir, elle a un sens puisqu'elle s'appuie sur un point déterminé : l'éthique. Fort de cette interprétation neuve de l'existence, "la vie devient belle", écrit l'assesseur de Kierkegaard dans l'Alternative (OC IV, 201 / II 241)

Cependant, il importe ici de préciser que le moi qui choisit l'éthique en lui n'est pas égoïste. S'il se choisit lui-même dans le temps, il ne fait jamais abstraction des autres. Car si le moi qui se choisit lui-même se donne une histoire individuelle, il n'en reste pas moins un moi en vivante interaction avec le monde réel auquel il appartient :

L'individu a donc en son moi une tâche (Opgave) proposée à son activité où il intervient comme personne précise dans les circonstances de la vie (...) Si l'individu ne s'est pas dès l'abord et foncièrement choisi comme personne concrète dans la continuité (concret personlighed i Continuitet), il ne saurait l'acquérir par la suite. S'il s'imagine que l'art consiste à commencer en Robinson, il reste toute sa vie un aventurier. Mais s'il voit que, faute d'un début concret, il ne parviendra jamais à commencer ni par conséquent à finir, il sera alors en continuité à la fois avec le passé et l'avenir. II se traduit de la vie personnelle en la vie sociale et inversement. (OCIV, 236/ II 284)

Si nous tirons les conséquences de cette lecture, nous reconnaissons que le mérite de l'éthique réside dans sa capacité de se «donner» sa conception du temps. L'homme de l'éthique connaît l'influence que le temps exerce sur lui, sur la sphère sociale, et s'il ne se donne pas naïvement au temps, il sait comment se produire lui-même à partir de son interprétation temporelle. Cette spécification au sujet de la conception adéquate du temps est décisive dans la mesure où elle permet à Kierkegaard (l'assesseur se confie ici au jeune homme de l'esthétique dans une lettre, seconde partie de Enten-Eller) d'illustrer deux attitudes erronées, maladives dira-t-il, face à la temporalité. Sous la plume d'un Kierkegaard fin psychologue et sensible à la maladie, nous retrouvons les intuitions de Pascal rapportées plus haut :

Toi qui as beaucoup observé, tu m'approuveras dans cette remarque générale que les hommes se répartissent en deux 
grandes catégories, suivant qu'ils vivent dans l'espérance ( $i$ håbet) ou dans le ressouvenir ( $i$ erindringen). Les deux catégories témoignent d'un rapport incorrect au temps (urigtig Forhold til Tiden). L'homme sain vit à la fois dans l'espérance et dans le ressouvenir, et c'est seulement de cette manière que sa vie prend une continuité vraie et pleine (hans liv sand, indholdsrig Continuiteerlighed). En revanche, je rappellerai que les individus ne vivent pas dans l'espérance seulement, mais que, pour eux, espérance et souvenir se compénètrent dans le temps présent. (OC IV, 128 / II 154-155. Notre traduction)

Kierkegaard précise ainsi que vouloir remonter le cours du temps ne sert à rien et que vouloir s'affranchir du temps non plus. S'il faut lire entre les lignes, ces attitudes sont malsaines, incorrectes (urigtige) et rendent malheureux. Car la santé viendra de la pleine continuité avec soi-même qui s'établit moins sur la négation d'une dimension temporelle par rapport aux autres que sur l'unification, plus précisément la compénétration des trois dimensions temporelles dans l'action pratique.

Résultant du choix de soi, la continuité s'avère une autotemporalisation qui change le passé déjà accompli en liberté en lui découvrant un sens pour le présent et l'avenir. Le temps n'est donc plus une forme vide comme chez Kant, mais une possibilité de devenir soi-même, assumée et réellement vécue. En prônant ainsi la continuité absolue de soi, Kierkegaard se trouve à défendre l'émergence du temps vécu comme mûrissement. Si l'esthétique s'expérimente au présent, dans un instant vide, le choix éthique "confère» un sens à tous ces moments épars dans l'action pratique, dans une histoire personnelle vécue dans le temps. L'assesseur peut alors répliquer au jeune homme qui défend la conception esthétique dans l'Alternative : "Tu as l'action pratique en horreur, et rien de plus naturel, car cette activité n'a de sens que si la vie possède la continuité qui manque à la tienne" (OC IV, 177 / II 212).

Le sens de la vie humaine ne se pense pas sans continuité. Celui qui refuse l'action pratique et ses exigences (surtout la continuité du sens que procure cette action), s'adonne au malheur. Kierkegaard, ce n'est vraiment pas surprenant, écrira à la fin de notre texte, "Le plus malheureux", ces lignes qui résument à elle seules le malheur : 
Seul et livré à lui-même dans le vaste monde, il n'a point de contemporanéité à laquelle se rattacher, point de passé après lequel soupirer, car son passé n'est pas encore venu, point d'avenir qu'il puisse espérer, car son avenir est déjà révolu. Dans sa solitude, il a devant lui, le monde entier comme le "tu» avec lequel il est en conflit; car le reste de monde n'est pour lui qu'une seule personne, et cette personne, cet ami, aussi inséparable qu'importun, c'est la méprise (OC IV, 211-212 / / 131)

Bien entendu, la question du malheur et du bonheur soulève la question du sens de la vie. L'homme malheureux ne connaîtra pas, du moins durant la période la plus sombre, la continuité et la contemporanéité du sens; il restera divisé et en conflit avec lui-même. La contemporanéité consiste justement à déchiffrer et à participer à un sens qui nous dépasse et qui nous transcende, c'est-à-dire à endre contemporain (donc présent) le sens d'un événement passé qui peut encore nous guider aujourd'hui. Catégorie chère à Kierkegaard («la pensée de sa vie» s'il faut en croire un passage du huitième numéro de I'Instant / OC XIX, 256), la contemporanéité exige une appropriation constante du sens. Est donc contemporain un événement dont la signification traverse le cours du temps pour venir nous parler personnellement. Dans l'extrait cité plus haut, Kierkegaard fait certainement allusion à la foi chrétienne dont le sens guide le chrétien de génération en génération 7 . Mais sur cette catégorie herméneutique de contemporanéité 8 , nous nous bornerons à ces quelques indications générales, car les développements philosophiques et théologiques qu'exige cette notion difficile nous mèneraient assurément trop loin. Pour le moment donc, gardons à l'esprit que c'est la continuité éthique, en tant qu'elle assure l'action pratique, qui "confère» ou "redonne" un sens à l'existence. II nous faut ici revenir au problème du malheur soulevé par Kierkegaard et qui est notre sujet principal.

\section{De la différence absolue entre le bonheur et le malheur}

Si l'homme veut éviter le malheur, laisse entendre notre texte, il doit s'efforcer, dans la pratique, à rattacher les instances du temps dans un présent signifiant pour lui. Ici, et c'est décisif pour la construction de l'histoire individuelle, il n'est pas tant question de la réalité vécue "réellement" par l'individu que de la manière dont il conçoit 
"sa» réalité. Autrement dit, ce n'est pas tant la temporalité réellement vécue qui importe lorsque l'on cherche le bonheur, de savoir par exemple s'il s'adonne au travail le lundi ou le jeudi, mais plutôt la conception que l'individu se fait lui-même de son propre temps de vie. Ainsi, il s'agit d'une interprétation subjective.

Cependant, même si l'interprétation est subjective, la conception éthique, comme agir, comme action dans le social, demeure la référence. Voilà pourquoi celui qui refuse l'action, c'est-à-dire l'accomplissement du devoir, refuse de mûrir son moi. Celui qui refuse les exigences de l'action restera par conséquent isolé, «seul contre le monde", dans son malheur. En ce sens, l'homme le plus malheureux se reconnaîtra, écrit Kierkegaard, en ce qu'il "craint la mort" (OC IV, 207). Pourquoi? En vertu de quoi la question du malheur rejoint-elle ici la mort?

C'est que la vie est comprise dialectiquement par Kierkegaard comme le contraire de la mort. II n'y pas de mort sans vie et inversement. La mort se «trouve" là où le sens de la vie n'est plus évident; la mort se ressent quand les possibilités de réaliser le moi idéal ont disparu et que tout devient indifférent. La mort, c'est cet inconfort surgissant de la "perte» du sens, c'est-à-dire lorsque le sens de la vie n'apparaît plus comme donné, où la continuité exigée par l'action pratique ne va plus de soi. Aux yeux de Kierkegaard, le désespoir est ce chemin vers la mort (ce que son pseudonyme Anti-Climcus explique rigoureusement dans un livre dont le titre n'est pas, en vérité, "le traité du désespoir» mais plutôt La maladie à la mort (Sygdommen til Døden)). La personne qui désespère, celle qui n'a plus d'espérance, n'a plus de vie, car elle n'a plus le goût de vivre : malade de la vie ou dans la vie pour mieux dire, elle se trouve, vivante, sur le chemin de la mort. Tout à l'opposé se trouve la personne qui se réalise, celle qui accomplit le sens de son existence et en ressent la plénitude. Celle-ci certes ne veut pas quitter la vie. Elle ne veut pas en effet quitter la vie précisément au sens où l'on dit que «la vie lui appartient". L'homme heureux, en paix avec lui-même (nous dirons désormais en accord avec "sa" conception du temps), vivra parmi les hommes, dans la situation psychologique opposée à celle du malheureux. Le malheureux (Den Ulykkelig), enfin, sera celui qui ne maîtrise pas son temps, qui n'a pas la maîtrise souhaitée de sa vie ou qui n'a pas, disons-le ainsi, cette chance (Ulykke).

Le lecteur attentif aura certes relevé un point décisif de cette explication au sujet des liens unissant la mort et le malheur. Face à la 
mort en effet, le bonheur et le malheur s'opposent diamétralement. L'expérience confirme souvent que la personne malheureuse ne peut comprendre la personne heureuse et inversement : leurs situations sont éloignées, peut-être incompatibles, du moins sur le plan du sens de l'existence. II n'en sera peut-être pas toujours ainsi. Mais dans leur différence absolue cependant, le sentiment de vide (ici associé au malheur) et le sentiment de plénitude (évidemment le bonheur) ont un point en commun et se doivent d'être rapprochés : ils défient le langage et notre capacité de dire. Ce n'est peut-être pas un hasard si les philosophes ont traditionnellement éprouvé tant de difficultés à "dire" ce qu'est le bonheur. Cela s'explique possiblement en vertu du fait que la personne parfaitement heureuse ne voit pas pourquoi ni comment elle mettrait en mots une expérience aussi singulière, aussi privilégiée et aussi personnelle, c'est-à-dire une expérience qui se sait elle-même être en rapport avec la chance d'être à la bonne place au bon moment. Tout à l'opposé, l'incapacité de dire est aussi partagée par la personne malheureuse. Le silence ici, et c'est bien ce qu'a compris Kierkegaard, convient peut-être davantage que le langage. Le silence est assurément la limite de toute éthique. Afin de rendre cette idée de l'absolue différence entre le bonheur et le malheur, il n'est peut-être pas déplacé de citer ici un extrait de "Coupable?" «Non coupable?» (un long texte aux accents autobiographiques qui constitue la partie religieuse des Stades sur le chemin de la vie, titre qui rappelle à quel point Kierkegaard s'était intéressé au sens dans l'existence), dans lequel l'auteur pseudonyme notait pertinemment :

Chose curieuse, malgré leur extrême différence, le bonheur et le malheur ont parfois un trait en commun de souhaiter le silence. (...) Rien ne doit séparer les amants ! Ah! Ah! Nous avons été unis (Régine et Kierkegaard) trop peu de temps pour cela - rien ne s'interpose entre nous, et cependant un monde nous sépare, un monde exactement. Par moments, je suis heureux, parfaitement heureux, plus heureux que je ne l'aie jamais rêvé, pourvu que j'y trouve une importante compensation à ma douleur... (OCIX, 175 et $200 / \mathrm{VI} 202$ et 229.

Le souligné est de SK)

Ce passage suggère un dernier point très important au sujet du bonheur dans la pensée de Kierkegaard. Si le malheur et le bonheur s'opposent radicalement et que le bonheur doit être envisagé dans son rapport à la douleur et la souffrance, c'est parce que l'expérience 
humaine relève essentiellement de la différence absolue, de l'incomplétude. En effet, pour Kierkegaard, l'homme demeure l'objet d'une synthèse à jamais différée d'éléments incommensurables, par exemple le corps et l'esprit, le fini et l'infini, le temps et l'éternité9. La différence des mondes est aussi, et peut-être davantage, celle du temps et de l'éternité. L'homme sait d'expérience que le bonheur terrestre ne dure pas, que le malheur appartient, comme la question du sens ou du télos de la vie, à la finitude, donc à l'immanence. Voilà pourquoi il doit prendre les moyens d'éviter, dans la mesure du possible, le malheur dont il est «lui-même» responsable, malheur qui est en fait sans équivalent avec le malheur que lui envoie Dieu comme épreuve (Cf. le récit de l'épreuve de Job dans La répétition). Ainsi, en attendant la béatitude éternelle, la joie ou la grâce, qui ne dépendent pas seulement de l'agir humain et qui sont sans doute plus proches du vrai bonheur, l'homme ordinaire s'assurera de vivre au présent dans l'éthique, parmi les hommes, en construisant son rapport au temps sur l'idée de continuité avec lui-même. Le bonheur terrestre, que Kierkegaard reconnaît avoir ressenti, demeurera sans équivalent avec la félicité éternelle, tout ce qui compte en bout de ligne pour lui.

\section{Dominic Desroches Département de philosophie Université de Montréal}

1. S. Kierkegaard, Post-scriptum définitif et non scientifique aux Miettes philosophiques, vol. II, Chap. III, $\S 4$. Nous référons désormais aux CEuvres complètes de Kierkegaard (OC), Trad. P-H. Tisseau, Éditions de l'Orante, Paris, 1966-1986. Nous ajoutons ensuite la référence à l'édition danoise de base des Kierkegaards Samlede Værker, Anden Udgave (SV 2), Copenhague, 1920-1936. Ici : OC XI, 51 / VII 340.

2. Sur la topologie existentielle, l'esthétique et le rapport au langage, nous renvoyons le lecteur à la première section de notre ouvrage qui paraîtra bientôt chez l'Harmattan : Expressions éthiques de l'intériorité. Coll. Ouverture philosophique, Paris, Harmattan, janvier 2004.

3. Voir ici $O C X \mathrm{XI}, 125$ / VII 423. Climacus écrit : «L'immédiateté est bonheur, car elle ne comporte pas de contradiction; l'homme de l'immédiat est par essence heureux, et la conception que l'immédiateté se fait de la vie est le bonheur. (...) En revanche, l'intériorité (l'individu éthique et éthico-religieux) voit dans la souffrance l'essentiel'.

4. $O C$ VI, 232-250, OC XI, 80-85 et OC XV, 85-151. À l'avenir, pour plus de commodité, les références aux $O C$ se trouveront dans le texte. 
5. A.H. Kahn «Happiness in Kierkegaards Efterskrifts», in Sophia, 1983, 22, № 1, 37-47.

6. G.W.F. Hegel, Grundlinien des Philosophie der Recht, Hamburg, Felix Meiner Verlag, 1955, trad. fr., Principes de la philosophie du droit, Gallimard, Paris, 1992.

7. On lira au sujet de la contemporanéité, les Miettes philosophiques et L'école du christinianisme: OC VII, 65 et OCXVII, 62-66).

8. Au passage, rappelons que Gadamer a repris et développé les catégories kierkegaardiennes de continuité et de contemporanéité dans son herméneutique philosophique, voir Wahrheit und Methode, Paul Siebeck, Tübingen, 1986, 126-139 / trad. fr. Vérité et méthode, Paris, Seuil, 1996, 139-149.

9. À ce sujet, on trouvera des précisions dans les Papirer et surtout Le concept d'angoisse (1844) et La maladie à la mort (1849) (OC XVI, 171) Sur cette question, lire entre autre Maurice Carignan, "L'éternel comme tiers synthétisant chez Kierkegaard", in Philosophiques, vol. VIII, N 1, 1981, 75-92. 